TRANSACTIONS OF THE

AMERICAN MATHEMATICAL SOCIETY

Volume 349, Number 8, August 1997, Pages 3391-3400

S 0002-9947(97)01882-5

\title{
EXTREME POINTS IN TRIANGULAR UHF ALGEBRAS
}

\author{
TIMOTHY D. HUDSON, ELIAS G. KATSOULIS, AND DAVID R. LARSON
}

\begin{abstract}
We examine the strongly extreme point structure of the unit balls of triangular UHF algebras. The semisimple triangular UHF algebras are characterized as those for which this structure is minimal in the sense that every strongly extreme point belongs to the diagonal. In contrast to this, for the class of full nest algebras we prove a Krein-Milman type theorem which asserts that every operator in the open unit ball of the algebra is a convex combination of strongly extreme points.
\end{abstract}

Results concerning the geometry of the unit ball have a long history both in Banach space theory and in the theory of operator algebras. This geometry can be affiliated with structural and algebraic properties. Moreover, differences in geometric properties can prove useful in classification problems. Two fundamental results are the Russo-Dye Theorem and Kadison's Theorem on isometries. More recently, there has been interest in the unit balls of nonselfadjoint operator algebras, especially nest algebras $[1,2,3,4,15,16]$.

This paper concerns the unit balls of triangular UHF algebras. These and the larger class of triangular AF algebras are nonselfadjoint analogues of the UHF and AF $\mathrm{C}^{*}$-algebras studied by Glimm and Bratteli. Their theory has grown rapidly, cf. $[8,9,18,19,20]$. We focus on the extreme point structure, and our results have a different flavor than those for nest algebras. Specifically, we study the strongly extreme points, those boundary points whose "stable character" with respect to approximations makes them behave well under direct limits. Triangular UHF algebras are direct limits of full upper triangular matrix algebras. Unit balls embed into unit balls in the direct limit scheme, and some types of embeddings respect the extreme point structure while others do not. This leads to structural differences in the limit algebras.

The geometric structures of the unit balls of different triangular UHF algebras can be very dissimilar. The convex hull of the strongly extreme points, even without closure, always contains the unit ball of the diagonal. Theorem 7 shows that the two coincide if and only if the algebra is semisimple. This is a characterization of a purely geometric property in terms of a purely algebraic one. In contrast to

Received by the editors January 11, 1996 and, in revised form, March 28, 1996.

1991 Mathematics Subject Classification. Primary 47D25, 46K50, 46B20.

Key words and phrases. Triangular operator algebra, strongly extreme point, UHF algebra, semisimple.

The first author's research was partially supported by NSF grant \#DMS-9500566 and the Linear Analysis and Probability Workshop at Texas A\&M University.

The second author's research was partially supported by a YI grant from the Linear Analysis and Probability Workshop at Texas A\&M University and a grant from East Carolina University.

The third author's research was partially supported by NSF grant \#DMS-9401544.

(C) 1997 American Mathematical Society 
this, Theorem 10 shows that the convex hull of the strongly extreme points of a triangular UHF algebra which is a full nest algebra always contains the open unit ball of the algebra.

A $\mathrm{C}^{*}$-algebra $\mathfrak{C}$ is approximately finite $(\mathrm{AF})$ if it is the norm-closed union of a nested sequence of finite-dimensional $\mathrm{C}^{*}$-subalgebras of $\mathfrak{C}$. If $\mathfrak{C}$ is unital and each of these finite-dimensional subalgebras contains the unit of $\mathfrak{C}$ and is isomorphic to some full matrix algebra $\mathbf{M}_{k}$, then $\mathfrak{C}$ is called uniformly hyperfinite (UHF). A normclosed subalgebra $\mathfrak{A}$ of $\mathfrak{C}$ is called triangular $A F$ (TAF) if its diagonal $\mathfrak{D}=\mathfrak{A} \cap \mathfrak{A}^{*}$ is a regular canonical masa in $\mathfrak{C}$ (see below).

The normalizer of $\mathfrak{D}$ in $\mathfrak{A}$ is

$$
\mathcal{N}_{\mathfrak{D}}(\mathfrak{A})=\left\{w \in \mathfrak{A} \mid w \text { is a partial isometry, } w \mathfrak{D} w^{*} \subseteq \mathfrak{D}, \text { and } w^{*} \mathfrak{D} w \subseteq \mathfrak{D}\right\} .
$$

Regular canonical masas are maximal abelian self-adjoint subalgebras of $\mathfrak{C}$ for which there exists a sequence of finite-dimensional $\mathrm{C}^{*}$-subalgebras of $\mathfrak{C}$, say $\left\{\mathcal{C}_{n}\right\}_{n}$, with $\mathfrak{C}=\overline{\bigcup_{n} \mathcal{C}_{n}}$, so that if $\mathcal{D}_{n}$ is $\mathcal{C}_{n} \cap \mathfrak{D}$, then $\mathfrak{D}=\overline{\bigcup_{n} \mathcal{D}_{n}}$ and for all $n, \mathcal{D}_{n}$ is a masa in $\mathcal{C}_{n}$ and $\mathcal{N}_{\mathcal{D}_{n}}\left(\mathcal{C}_{n}\right) \subseteq \mathcal{N}_{\mathcal{D}_{n+1}}\left(\mathcal{C}_{n+1}\right)$, where we define $\mathcal{N}_{\mathfrak{D}_{k}}\left(\mathfrak{C}_{k}\right)$ analogously to $\mathcal{N}_{\mathfrak{D}}(\mathfrak{A})$. If $\mathcal{A}_{n}$ is $\mathcal{C}_{n} \cap \mathfrak{A}$ and $\varphi_{n}$ is the inclusion from $\mathcal{A}_{n}$ to $\mathcal{A}_{n+1}$, then $\mathfrak{A}=\overline{\bigcup_{n} \mathcal{A}_{n}}$ and (i) each $\mathcal{A}_{n}$ is triangular in $\mathcal{C}_{n}$, (ii) each $\varphi_{n}$ extends to a $*$-homomorphism from $\mathcal{C}_{n}$ to $\mathcal{C}_{n+1}$, and (iii) for each $n$, the extension of $\varphi_{n}$ maps $\mathcal{N}_{\mathcal{D}_{n}}\left(\mathcal{C}_{n}\right)$ into $\mathcal{N}_{\mathcal{D}_{n+1}}\left(\mathcal{C}_{n+1}\right)$. Then $\mathfrak{A}$ is isometrically isomorphic to the inductive limit of the system

$$
\mathcal{A}_{1} \stackrel{\varphi_{1}}{\longrightarrow} \mathcal{A}_{2} \stackrel{\varphi_{2}}{\longrightarrow} \mathcal{A}_{3} \stackrel{\varphi_{3}}{\longrightarrow} \mathcal{A}_{4} \cdots \text {. }
$$

Conversely, if we have algebras $\left\{\mathcal{A}_{n}\right\}_{n}$ and $\left\{\mathcal{C}_{n}\right\}_{n}$ with $\mathcal{A}_{n} \subseteq \mathcal{C}_{n}$ and injective maps $\varphi_{n}: \mathcal{A}_{n} \rightarrow \mathcal{A}_{n+1}$ that satisfy the three properties listed above, then the inductive limit of (1) is a triangular AF algebra. We will denote this inductive limit by $\lim \left(\mathcal{A}_{n}, \varphi_{n}\right)$ and call (1) a presentation for the inductive limit. For $k \leq n$, let $\varphi_{n, k}: \overrightarrow{\mathcal{A}_{k}} \rightarrow \mathcal{A}_{n}$ be the composition $\varphi_{n-1} \circ \cdots \circ \varphi_{k}$. By a triangular UHF algebra, we will mean a TAF algebra that is the direct limit of a system as in (1), where $\mathcal{A}_{n}$ is isometrically isomorphic to some full upper triangular matrix algebra $\mathbf{T}_{p_{n}}$ and $\varphi: \mathcal{A}_{n} \rightarrow \mathcal{A}_{n+1}$ is an embedding satisfying properties (ii) and (iii) above. We shall make the usual identification of $\mathrm{C}^{*}\left(\mathcal{A}_{n}\right)$ with $\mathbf{M}_{p_{n}}$.

Since for each $n \mathcal{D}_{n}$ is a masa in $\mathcal{C}_{n}$, we can choose a system of matrix units for each $\mathcal{C}_{n}$ so that those for $\mathcal{C}_{n}$ are sums of those for $\mathcal{C}_{n+1}$, and the self-adjoint ones are in $\mathcal{D}_{n}$ [20]. Throughout the paper, we fix such a system for $\mathfrak{A}=\lim \left(\mathcal{A}_{n}, \varphi_{n}\right)$. Since a matrix unit in $\mathcal{A}_{n}$ is in $\mathcal{N}_{\mathcal{D}_{n}}\left(\mathcal{A}_{n}\right)$ and $\varphi_{m}\left(\mathcal{N}_{\mathcal{D}_{m}}\left(\mathcal{A}_{m}\right)\right) \subseteq \mathcal{N}_{\mathcal{D}_{m+1}}\left(\mathcal{A}_{m+1}\right)$ for every $m$, all such matrix units are in $\mathcal{N}_{\mathfrak{D}}(\mathfrak{A})$. If $e$ is a matrix unit in $\mathcal{A}_{n}$ and $p$ is a projection in $\mathfrak{D}$ with $p e \neq 0$, then we call pe a restriction or subordinate of $e$. Let $\mathcal{P}(\mathfrak{A})$ denote the collection of all diagonal projections of $\mathfrak{A}$. The diagonal order [18] on $\mathcal{P}(\mathfrak{A})$, denoted by “ฏ”, is a partial order given by

$$
e \preceq f \Longleftrightarrow \text { there exists } w \in \mathcal{N}_{\mathfrak{D}}(\mathfrak{A}) \text { with } w w^{*}=e, w^{*} w=f \text {. }
$$

If $e \preceq f$ and $e \neq f$, then we write $e \prec f$. For a partial isometry $p$ in $\mathfrak{A}$, let $d(p)$ denote the domain projection $p^{*} p$ and $r(p)$ the range projection $p p^{*}$.

We will use the following result of Moore and Trent [15].

Lemma 1. Let $A$ be a contraction in $\mathcal{B}(\mathcal{H})$, where $\mathcal{H}$ is a Hilbert space, and define

$$
G_{A}=\left(I-\left(A^{*} A\right)^{\frac{1}{2}}\right)^{\frac{1}{2}} \quad \text { and } \quad G_{A^{*}}=\left(I-\left(A A^{*}\right)^{\frac{1}{2}}\right)^{\frac{1}{2}} .
$$

If $X \in \mathcal{B}(\mathcal{H})$ and $\|X\| \leq 1$, then $\left\|A \pm G_{A^{*}} X G_{A}\right\| \leq 1$. 
Let $\mathfrak{X}_{1}$ denote the closed unit ball of the Banach space $\mathfrak{X}$. For $x \in \mathfrak{X}_{1}$ and $\epsilon>0$, we define

$$
\delta(x ; \epsilon)=\sup \left\{\|z\| \mid z \in \mathfrak{X}_{1},\|x \pm z\| \leq 1+\epsilon\right\},
$$

and

$$
\delta(x)=\lim _{\epsilon \downarrow 0} \delta(x ; \epsilon) .
$$

Definition 2. If $x$ is an element of $\mathfrak{X}_{1}$, we say $x$ is strongly extreme if $\delta(x)=$ 0 . (This is an equivalent formulation of the definition used in [7, p. 105].)

Strongly extreme points have been studied in the literature. Cima and Thomson proved that the strongly extreme points in $\mathrm{H}^{\infty}$ are the inner functions [5]. Davidson, Feeman, and Shields studied extreme points in quotients of operator algebras and considered strongly extreme points [6]. Other authors have studied strongly extreme points in Banach spaces $[7,12,14]$.

We will write $\operatorname{ext}(K)$ for the set of extreme point of the convex set $K$ and s$\operatorname{ext}(K)$ for the strongly extreme points of $K$. We write $\overline{\mathrm{co}}(\cdot)$ for the closed convex hull.

We thank Dan Timotin for the following example.

Example 3. Let $\mathcal{X}=\bigoplus_{p=1}^{\infty} \ell^{p}(2)$, and set $x=(1,0) \oplus(1,0) \oplus \ldots$ For each $n=1,2, \ldots$, define

$$
x_{n}=\underbrace{(0,0) \oplus \cdots \oplus(0,0) \oplus(0,1)}_{n \text { entries }} \oplus(0,0) \oplus \cdots
$$

Then $x$ is an extreme point of $\mathcal{X}_{1}$ since $(1,0)$ is an extreme point in $\left(\ell^{p}(2)\right)_{1}$. However, $\lim _{n}\left\|x \pm x_{n}\right\|=1$. Thus, $\delta(x ; \epsilon) \geq 1$ for all $\epsilon>0$, and so $x$ is not strongly extreme.

Lemma 4. If $\mathfrak{A}$ is a TAF algebra, then the convex hull (no closure) of the strongly extreme points in $\mathfrak{A}_{1}$ contains $\mathfrak{D}_{1}$.

Proof. By [6, Lemma 2] it follows that every unitary in $\mathfrak{A}$ is a strongly extreme point. The conclusion now follows from the strong version of the Russo-Dye Theorem in [22].

The Jacobson radical of a Banach algebra is the maximal topologically nil ideal in the algebra. A Banach algebra is called semisimple if its Jacobson radical is zero. Donsig characterized semisimplicity for TAF algebras in terms of mixing embeddings [8, Corollary 6]. If $\varphi: \mathcal{A}_{n} \rightarrow \mathcal{A}_{n+1}$ is an embedding and $p$ and $q$ are projections in $\mathbf{D}_{p_{n}}$ such that $p \preceq q$, then $\varphi$ mixes $p$ and $q$ if there are restrictions $r$ of $\varphi(p)$ and $s$ of $\varphi(q)$ such that $s \preceq r$. If $\varphi$ mixes $p$ and $q$ for every such $p$ and $q$ in $\mathbf{D}_{p_{n}}$, then the embedding $\varphi$ is called mixing. Donsig proved that a triangular UHF algebra is semisimple if and only if it has a presentation for which all embeddings are mixing. The standard and alternation limit algebras are semisimple. See [8] for other examples.

The following proposition is a key result.

Proposition 5. Let $\mathfrak{A}$ be a semisimple triangular UHF algebra.

If $A \in \mathfrak{A}_{1}$, then $\delta(A) \geq\left\|G_{A}\right\|\left\|G_{A^{*}}\right\|$.

Proof. Since $\mathfrak{A}$ is semisimple, by $[8$, Corollary 6], we can choose a presentation $\underset{\lim }{\longrightarrow}\left(\mathcal{A}_{n} ; \varphi_{n}\right)$ for $\mathfrak{A}$, where each $\varphi_{n}: \mathcal{A}_{n} \rightarrow \mathcal{A}_{n+1}$ is mixing and $\mathcal{A}_{n}$ is isometrically 
isomorphic to $\mathbf{T}_{p_{n}}$ for some $n$. Choose a system of matrix units for $\mathfrak{A}$ so that for each $n,\left\{e_{i, j}^{(n)}: 1 \leq i \leq j \leq p_{n}\right\}$ is a system of matrix units for $\mathcal{A}_{n}$. Since the embeddings $\varphi_{n}$ are $*$-extendible, they may also be viewed as mappings on $\mathbf{M}_{p_{n}}$.

Let $\epsilon>0$ be given and let $A \in \mathfrak{A}_{1}$. There exists $N \geq 1$ such that $\operatorname{dist}\left(A, \mathcal{A}_{n}\right)<\epsilon$ for all $n \geq N$. For each $n \geq N$, choose $A_{n} \in \mathcal{A}_{n}$ with $\left\|A-A_{n}\right\|<\epsilon$. We first construct operators $X_{n} \in \mathcal{A}_{n+1}$ whose norm is approximately $\left\|G_{A}\right\|\left\|G_{A^{*}}\right\|$ such that $\left\|X_{n} \pm A_{n}\right\| \leq 1$. Fix $n \geq N$.

We begin by defining operators $Y_{Z}$ in $\mathbf{M}_{p_{n+1}}$ for specific choises of $Z$ in $\mathbf{M}_{p_{n}}$.

First notice that since $\varphi_{n}$ is mixing there exist restrictions $e_{i_{p_{n}}, i_{p_{n}}}^{(n+1)}$ of $\varphi_{n}\left(e_{p_{n}, p_{n}}^{(n)}\right)$ and $e_{j_{1}, j_{1}}^{(n+1)}$ of $\varphi_{n}\left(e_{1,1}^{(n)}\right)$ so that $e_{i_{p_{n}}, i_{p_{n}}}^{(n+1)} \prec e_{j_{1}, j_{1}}^{(n+1)}$, i.e., $i_{p_{n}}<j_{1}$. Using the fact that $\varphi_{n}$ maps matrix units to sums of matrix units, we conclude that there are diagonal matrix units $e_{i_{k}, i_{k}}^{(n+1)}, k=1, \ldots, p_{n}-1$, and $e_{j_{l}, j_{l}}^{(n+1)}, l=2, \ldots, p_{n}$, which are defined recursively as follows:

$$
\begin{aligned}
& e_{i_{k}, i_{k}}^{(n+1)}=\left(\varphi_{n}\left(e_{k, k+1}^{(n)}\right) e_{i_{k+1}, i_{k+1}}^{(n+1)}\right)\left(\varphi_{n}\left(e_{k, k+1}^{(n)}\right) e_{i_{k+1}, i_{k+1}}^{(n+1)}\right)^{*}, \\
& e_{j_{l}, j_{l}}^{(n+1)}=\left(e_{j_{l-1}, j_{l-1}}^{(n+1)} \varphi_{n}\left(e_{l-1, l}^{(n)}\right)\right)^{*}\left(e_{j_{l-1}, j_{l-1}}^{(n+1)} \varphi_{n}\left(e_{l-1, l}^{(n)}\right)\right) .
\end{aligned}
$$

Assume that $Z$ is some contraction in $\mathbf{M}_{p_{n}}$ and write $Z=\left[z_{i j}\right]_{1 \leq i, j \leq p_{n}}$. Define $Y_{Z}$ to be the element of $\mathbf{M}_{p_{n+1}}$ so that

$$
e_{r, r}^{(n+1)} Y_{Z} e_{s, s}^{(n+1)}= \begin{cases}z_{k, l} & \text { if } r=i_{k} \text { and } s=j_{l}, \\ 0 & \text { otherwise }\end{cases}
$$

for $1 \leq r, s \leq p_{n+1}$.

Two remarks are in order. First, all of the partial isometries $\varphi_{n}\left(e_{k, k+1}^{(n)}\right), k=$ $1, \ldots, p_{n}-1$, and $\varphi_{n}\left(e_{l-1, l}^{(n)}\right), l=2, \ldots, p_{n}$, are in $\mathcal{A}_{n+1}$, and so the collections $\left(i_{k}\right)_{k=1}^{p_{n}}$ and $\left(j_{l}\right)_{l=1}^{p_{n}}$ are increasing. Second, from the definition of $e_{i_{k}, i_{k}}^{(n+1)}$ it follows that $e_{i_{k}, i_{k}}^{(n+1)} \varphi_{n}\left(e_{s, t}^{(n)}\right) e_{i_{k^{\prime}}, i_{k^{\prime}}}^{(n+1)}$ is nonzero only in the case where $s=k$ and $t=k^{\prime}$, and in this case $e_{i_{k}, i_{k}}^{(n+1)} \varphi_{n}\left(e_{s, t}^{(n)}\right) e_{i_{k^{\prime}}, i_{k^{\prime}}}^{(n+1)}$ is a matrix unit restriction of $e_{s, t}^{(n)}$ in $\mathbf{M}_{p_{n+1}}$. Thus, given an arbitrary $a=\sum_{s, t=1}^{p_{n}} a_{s, t} e_{s, t}^{(n)}$ in $\mathbf{M}_{p_{n}}$, we have

$$
\left(\sum_{k=1}^{p_{n}} e_{i_{k}, i_{k}}^{(n+1)}\right) \varphi_{n}(a)\left(\sum_{k^{\prime}=1}^{p_{n}} e_{i_{k^{\prime}}, i_{k^{\prime}}}^{(n+1)}\right)=\sum_{s, t=1}^{p_{n}} a_{s, t}\left(e_{i_{s}, i_{s}}^{(n+1)} \varphi_{n}\left(e_{s, t}^{(n)}\right) e_{i_{t}, i_{t}}^{(n+1)}\right) .
$$

A similar statement is also valid for the matrix units $e_{j_{l}, j_{l}}^{(n+1)}, l=1, \ldots, p_{n}$.

It follows from the first remark above that $Y_{Z}$ belongs to $\mathcal{A}_{n+1}$ and $\left\|Y_{Z}\right\|=\|Z\|$. The second one implies that there exist a unitary $U$ in $\mathrm{C}^{*}\left(\mathcal{A}_{n+1}\right)$ (corresponding to an interchange of rows and columns), a projection $e$ belonging to Lat $\mathcal{A}_{n+1} \cap\{U\}^{\prime}$ (actually, $e=\sum_{s=1}^{i_{p_{n}}} e_{s, s}^{(n+1)}$ ), and projections $p, q \in \mathcal{A}_{n+1}$ satisfying $p \leq e \leq q \leq 1$ so that, for any $S \in \mathbf{M}_{p_{n}}$,

$$
U^{*} \varphi_{n}(S) U=\left[\begin{array}{cccc}
S & 0 & 0 & 0 \\
0 & * & 0 & * \\
0 & 0 & S & 0 \\
0 & 0 & 0 & *
\end{array}\right]
$$


and also

$$
U^{*} Y_{Z} U=\left[\begin{array}{cccc}
0 & 0 & Z & 0 \\
0 & 0 & 0 & 0 \\
0 & 0 & 0 & 0 \\
0 & 0 & 0 & 0
\end{array}\right]
$$

with respect to the decomposition $p \oplus(e-p) \oplus(q-e) \oplus(1-q)=1$.

Let $Z_{n}$ be a suitable rank one operator in $\mathbf{M}_{p_{n}}$ so that

$$
\left\|G_{A_{n}} Z_{n} G_{A_{n}^{*}}\right\|=\left\|G_{A_{n}}\right\|\left\|G_{A_{n}^{*}}\right\|,
$$

and let

$$
X_{n}=\left(I-\left(\varphi_{n}\left(A_{n}^{*} A_{n}\right)\right)^{\frac{1}{2}}\right)^{\frac{1}{2}} Y_{Z_{n}}\left(I-\left(\varphi_{n}\left(A_{n} A_{n}^{*}\right)\right)^{\frac{1}{2}}\right)^{\frac{1}{2}} .
$$

By Lemma 1 we have that $\left\|X_{n} \pm \varphi_{n}\left(A_{n}\right)\right\| \leq 1$, and so $\left\|X_{n} \pm A\right\| \leq 1+\epsilon$.

It remains to show that $X_{n}$ belongs to $\mathcal{A}_{n+1}$. Notice that

$$
\begin{aligned}
U^{*} X_{n} U & =U^{*} \varphi_{n}\left(G_{A_{n}}\right) Y_{Z_{n}} \varphi_{n}\left(G_{A_{n}^{*}}\right) U \\
& =\left(U^{*} \varphi_{n}\left(G_{A_{n}}\right) U\right)\left(U^{*} Y_{Z_{n}} U\right)\left(U^{*} \varphi_{n}\left(G_{A_{n}^{*}}\right) U\right) \\
& =\left[\begin{array}{cccc}
0 & 0 & G_{A_{n}} Z_{n} G_{A_{n}^{*}} & 0 \\
0 & 0 & 0 & 0 \\
0 & 0 & 0 & 0 \\
0 & 0 & 0 & 0
\end{array}\right] .
\end{aligned}
$$

Since $e$ commutes with $U$, we conclude that $X_{n}=e X_{n} e^{\perp}$, and so $X_{n}$ belongs to $\mathcal{A}_{n+1}$. Hence,

$$
\delta(A ; \epsilon) \geq\left\|X_{n}\right\|=\left\|G_{A_{n}} Z_{n} G_{A_{n}^{*}}\right\|=\left\|G_{A_{n}}\right\|\left\|G_{A_{n}^{*}}\right\| .
$$

Taking limits, we obtain $\delta(A) \geq\left\|G_{A}\right\|\left\|G_{A^{*}}\right\|$, as desired.

Lemma 6. Let $\varphi: \mathbf{T}_{n} \rightarrow \mathbf{T}_{m}$ be an embedding which is not mixing, $x=\sum_{i=1}^{n-1} e_{i, i+1}^{(n)}$, and let $0<\epsilon<1$. Then, in $\mathbf{T}_{m}, \delta(\varphi(x) ; \epsilon) \leq \sqrt{6 \epsilon}$.

Proof. Suppose that $\varphi$ is not mixing. Then, in fact, $\varphi$ fails to mix the pair $e_{1,1}^{(n)}$ and $e_{n, n}^{(n)}$. It follows that there exists $1 \leq j \leq m$ so that $\varphi\left(e_{1,1}^{(n)}\right) \leq \sum_{i=1}^{j} e_{i, i}^{(m)}$ and $\varphi\left(e_{n, n}^{(n)}\right) \leq I-\sum_{i=1}^{j} e_{i, i}^{(m)}$. Let $e=\sum_{i=1}^{j} e_{i, i}^{(m)}$. Let $z$ be an element of $\mathbf{T}_{m}$ so that $\|\varphi(x) \pm z\| \leq 1+\epsilon$. Then

$$
\left\|(\varphi(x) \pm z)^{*}(\varphi(x) \pm z)\right\| \leq(1+\epsilon)^{2} \leq 1+3 \epsilon,
$$

and so $\varphi(x)^{*} \varphi(x)+z^{*} z+\varphi(x)^{*} z+z^{*} \varphi(x) \leq(1+3 \epsilon) I$ and $\varphi(x)^{*} \varphi(x)+z^{*} z-$ $\varphi(x)^{*} z-z^{*} \varphi(x) \leq(1+3 \epsilon) I$. Thus,

$$
z^{*} z \leq I-\varphi(x)^{*} \varphi(x)+3 \epsilon I=\varphi\left(e_{1,1}^{(n)}\right)+3 \epsilon I .
$$

Hence, $(1-e) z^{*} z(1-e) \leq 3 \epsilon(1-e)$, and so $\|z(1-e)\| \leq \sqrt{3 \epsilon}$. Similarly, using the inequality $\left\|(\varphi(x) \pm z)(\varphi(x) \pm z)^{*}\right\| \leq 1+3 \epsilon$, one obtains $z z^{*} \leq I-\varphi(x) \varphi(x)^{*}+3 \epsilon I=$ $\varphi\left(e_{n, n}^{(n)}+3 \epsilon I\right.$, and so $\|e z\| \leq \sqrt{3 \epsilon}$. Since $e$ is invariant for $\mathbf{T}_{m}$, then

$$
\|z\|=\|z(1-e)+e z e\| \leq\left(\|z(1-e)\|^{2}+\|e z\|^{2}\right)^{\frac{1}{2}} \leq \sqrt{6 \epsilon},
$$

and the conclusion follows. 
Recall from [8] that if $x \in \mathcal{N}_{\mathfrak{D}}(\mathfrak{A})$, then $s \in \mathcal{N}_{\mathfrak{D}}(\mathfrak{A})$ is a link for $x$ if $s \neq 0, r(s)$ is a subprojection of $d(x)$, and $d(s)$ is a subprojection of $r(x)$.

Theorem 7. Let $\mathfrak{A}$ be a triangular UHF algebra. Then $\mathfrak{A}$ is semisimple if and only if every strongly extreme point of $\mathfrak{A}_{1}$ belongs to the diagonal $\mathfrak{D}$. In this case, the convex hull of the strongly extreme points of $\mathfrak{A}_{1}$ coincides with $\mathfrak{D}_{1}$.

Proof. ( $\Longrightarrow)$ First assume that $\mathfrak{A}$ is semisimple, and let $A \in \operatorname{s-ext}\left(\mathfrak{A}_{1}\right)$. Proposition 5 shows that $A^{*} A=I$ or $A A^{*}=I$. The existence of a faithful trace on $\mathrm{C}^{*}(\mathfrak{A})$ now shows that $A A^{*}=A^{*} A=I$, and so $A$ is unitary. Since $\mathfrak{A}$ is a direct limit of inverse closed algebras, then $\mathfrak{A}$ is inverse closed. Thus, $A \in \mathfrak{D}$.

$(\Longleftarrow)$ Assume that $\mathfrak{A}$ is not semisimple, and choose a presentation $\lim \left(\mathcal{A}_{n} ; \varphi_{n}\right)$ for $\mathfrak{A}$. We claim there exists some positive integer $m$ such that none of the maps $\varphi_{n, m}$, for any $n \geq m$, are mixing. Indeed, since $\mathfrak{A}$ is not semisimple, then by [8, Theorem 4] there is an off-diagonal matrix unit $g$ in $\mathfrak{A}$ with no links. Suppose $g$ belongs to $\mathcal{A}_{m}$ for some $m$, and let $n \geq m$. Let $e$ be the range projection of $g$ and $f$ the domain projection; hence $e \prec f$. If $\varphi_{n, m}$ were mixing, then there would be restrictions $r$ of $e$ and $s$ of $f$ in $\mathcal{A}_{n}$ so that $s \prec r$. Let $p$ be the matrix unit such that $p p^{*}=s$ and $p^{*} p=r$. Since $g g^{*}=e$ and $g^{*} g=f$, it follows that $p$ is a link for $g$, a contradiction.

Now apply Lemma 6 to each embedding $\varphi_{n, m}: \mathcal{A}_{m} \rightarrow \mathcal{A}_{n}$, and view these embeddings as inclusions. Conclude that there is a non-diagonal element $x$ of $\mathcal{A}_{m}$ so that for any $0<\epsilon<1$, if $z \in\left(\bigcup_{n=m}^{\infty} \mathcal{A}_{n}\right)_{1}$ with $\|x \pm z\| \leq 1+\epsilon$, then $\|z\| \leq \sqrt{6 \epsilon}$. It follows that $\delta(x ; \epsilon) \leq 2 \sqrt{6 \epsilon}$. So $x$ is strongly extreme in $\mathfrak{A}_{1}$, contradicting the hypothesis.

The last statement is immediate from Lemma 4.

Question. Let $\mathcal{X}$ be the direct limit of a system of the form

$$
\mathcal{X}_{1} \stackrel{\varphi_{1}}{\longrightarrow} \mathcal{X}_{2} \stackrel{\varphi_{2}}{\longrightarrow} \mathcal{X}_{3} \stackrel{\varphi_{3}}{\longrightarrow} \mathcal{X}_{4} \cdots
$$

where each $\mathcal{X}_{n}$ is isometrically isomorphic to some strictly upper triangular matrix algebra $\operatorname{rad} \mathbf{T}_{p_{n}}$ and $\varphi_{n}$ is mixing. Then an adaptation of the proof of Proposition 5 shows that the unit ball of $\mathcal{X}$ fails to have any strongly extreme points. Does the unit ball of $\mathcal{X}$ have any extreme points?

The triangular UHF algebra $\lim \left(\mathcal{A}_{n}, \varphi_{n}\right)$ is called a full nest algebra if each embedding $\varphi_{n}$ is a nest embedding, i.e., if for every $n, \varphi_{n}\left(\right.$ Lat $\left.\mathcal{A}_{n}\right) \subseteq$ Lat $\mathcal{A}_{n+1}$. If $\varphi: \mathbf{T}_{k} \rightarrow \mathbf{T}_{n k}$ is a nest embedding, then there is a unitary $U$ in the commutant of $\varphi\left(\operatorname{LatT}_{n k}\right)$ so that $\varphi(S)=U^{*}\left(S \otimes I_{n}\right) U$, where $I_{n}$ is the $n \times n$ identity matrix (indeed, using the notation of Power [20, p. 39], $U$ will be the block diagonal unitary $\left.\operatorname{diag}\left(I_{n}, U_{12}, U_{13}, \ldots, U_{1 k}\right)\right)$. These algebras are nest subalgebras of their enveloping $\mathrm{C}^{*}$-algebras. The refinement and twist limit algebras are full nest algebras; the term comes from [10].

Lemma 8. If $\mathfrak{A}$ is a full nest algebra, then the convex hull of the strongly extreme points of $\mathfrak{A}_{1}$ is dense in $\mathfrak{A}_{1}$.

Proof. Since the union of $\left(\mathfrak{A}_{n}\right)_{1}$ is dense in $\mathfrak{A}_{1}$ (regarding the embeddings as inclusions), then, using the Krein-Milman Theorem, it is enough to show that the 
extreme points of the unit ball of $\mathcal{A}_{n}, n=1,2, \ldots$, are strongly extreme in $\mathfrak{A}_{1}$. (It is not obvious even that they need be extreme.) So let $A \in \operatorname{ext}\left(\mathcal{A}_{n}\right)_{1}$. By way of contradiction, assume that there exist a constant $M>0$ and a sequence $\left\{X_{k}\right\}_{k=1}^{\infty}$, with $X_{k} \in \mathcal{A}_{n_{k}}$, so that

$$
\left\|\varphi_{n_{k}, n}(A) \pm X_{k}\right\| \leq 1+\frac{1}{k}
$$

and $\left\|X_{k}\right\| \geq M$ for $k \geq 1$. Since each $\varphi_{n_{k}, n}$ is a nest embedding, there exists a unitary $U_{k}$ in the commutant of $\varphi_{n_{k}, n}\left(\right.$ Lat $\left.\mathcal{A}_{n}\right)$ so that $\varphi_{n_{k}, n}(S)=U_{k}^{*}(S \otimes I) U_{k}$ for all $S$. Thus, $\mathrm{C}^{*}\left(\mathcal{A}_{n_{k}}\right)$ factors as $\mathrm{C}^{*}\left(\mathcal{A}_{n}\right) \otimes \mathbf{M}_{s_{k}}$, for some $s_{k} \in \mathbb{N}$, and $\varphi_{n_{k}, n}$ acts by inflating $S$ and then composing $S \otimes I$ with the block diagonal unitaries $U_{k}$ and $U_{k}^{*}$.

For each $k=1,2, \ldots$, there exist states $\phi_{k}$ and $\psi_{k}$ on $\mathrm{C}^{*}\left(\mathcal{A}_{n}\right)$ and $\mathbf{M}_{s_{k}}$, respectively, so that

$$
\left|\phi_{k} \otimes \psi_{k}\left(U_{k} X_{k} U_{k}^{*}\right)\right| \geq\left(\operatorname{dim} \mathrm{C}^{*}\left(\mathcal{A}_{n}\right)\right)^{-1} M .
$$

This last statement is obtained by using the fact that if $K$ is some positive constant, $A$ belongs to $\mathbf{M}_{m} \otimes \mathcal{B}(\mathcal{H})$, and $|(\rho \otimes \sigma)(A)| \leq K$ for all positive vector states $\rho$ and $\sigma$ of norm one, then $\|A\| \leq m^{2} K$.

Let $\Phi_{k}$ be the left slice map from $\mathrm{C}^{*}\left(\mathcal{A}_{n}\right) \otimes \mathbf{M}_{s_{k}}$ to $\mathrm{C}^{*}\left(\mathcal{A}_{n}\right)$ determined by $\psi_{k}$. So $\rho\left(\Phi_{k}(X)\right)=\rho \otimes \psi_{k}(X)$ for all $\rho \in\left(\mathrm{C}^{*}\left(\mathcal{A}_{n}\right)\right)_{*}, X \in \mathrm{C}^{*}\left(\mathcal{A}_{n}\right) \otimes \mathbf{M}_{s_{k}}$. Then

$$
\Phi_{k}((S \otimes I) X(T \otimes I))=S \Phi_{k}(X) T
$$

for each $X$ in $\mathrm{C}^{*}\left(\mathcal{A}_{n}\right) \otimes \mathbf{M}_{s_{k}}$ and all $S$ and $T$ in $\mathrm{C}^{*}\left(\mathcal{A}_{n}\right)$ (cf. [13, Problem 12.4.36]).

Now notice that $U_{k} X_{k} U_{k}^{*}$ leaves invariant every element of $\left(\right.$ Lat $\left.\mathcal{A}_{n}\right) \otimes I$, and so (2) implies that $\Phi_{k}\left(U_{k} X_{k} U_{k}^{*}\right)$ belongs to $\mathcal{A}_{n}$. Thus,

$$
\begin{aligned}
\left\|A \pm \Phi_{k}\left(U_{k} X_{k} U_{k}^{*}\right)\right\| & =\left\|\Phi_{k}\left(A \otimes I \pm U_{k} X_{k} U_{k}^{*}\right)\right\| \\
& \leq\left\|A \otimes I \pm U_{k} X_{k} U_{k}^{*}\right\| \\
& =\left\|U_{k}^{*}(A \otimes I) U_{k} \pm X_{k}\right\| \leq 1+\frac{1}{k}
\end{aligned}
$$

and $\left\|\Phi_{k}\left(U_{k} X_{k} U_{k}^{*}\right)\right\| \geq\left[\operatorname{dim} \mathrm{C}^{*}\left(\mathcal{A}_{n}\right)\right]^{-1} M$. This shows that $A$ is not strongly extreme (and thus, not extreme) in $\mathcal{A}_{n}$, a contradiction.

Lemma 9. If $\mathfrak{A}$ is a full nest algebra, then the convex hull of the strongly extreme points in $\mathfrak{A}_{1}$ contains some open ball centered at zero.

Proof. Since $\mathfrak{A}$ is a full nest algebra, there exists an increasing sequence $\left\{\mathcal{C}_{n}\right\}_{n=1}^{\infty}$ of finite dimensional factors with $\mathrm{C}^{*}(\mathfrak{A})=\overline{\bigcup_{n=1}^{\infty} \mathcal{C}_{n}}$, and a nest $\mathcal{N}$ in $\mathrm{C}^{*}(\mathfrak{A})$ so that if $\mathcal{N}_{n}=\mathcal{N} \cap \mathcal{C}_{n}$, then $\mathcal{A}_{n}=\operatorname{Alg} \mathcal{N}_{n} \cap \mathcal{C}_{n}$ is a maximal triangular subalgebra of $\mathcal{C}_{n}$ and $\mathfrak{A}=\operatorname{Alg} \mathcal{N}=\overline{\bigcup_{n=1}^{\infty} \mathcal{A}_{n}}$.

Hence, without loss of generality we may assume that $\mathcal{N}_{1}$ has $m$ elements, where $m \geq 4$. Let $\left\{e_{i, j}\right\}_{i, j=1}^{m}$ be a matrix unit system for $\mathcal{C}_{1}$ so that $\mathcal{N}_{1}=$ $\left\{\sum_{i=1}^{k} e_{i, i} \mid 1 \leq k \leq m\right\}$. We will show that the convex hull of the strongly extreme points, co $\left(\operatorname{s-ext}\left(\mathfrak{A}_{1}\right)\right)$, contains the sphere with center 0 and radius $a^{-1}$, where $a=\frac{1}{2} m(m+1)$.

Let $A \in \mathfrak{A}$ with $\|A\|<a^{-1}$ and let $a_{i, j}=a e_{i, i} A e_{j, j}$. Then each $a_{i, j}$ has norm strictly less than 1 , at most $a=\frac{1}{2} m(m+1)$ of the $a_{i j}$ are nonzero, and, moreover,

$$
A=\frac{1}{a} \sum_{1 \leq i \leq j \leq m} a_{i, j}
$$


Thus, it suffices to show that each $a_{i, j}$ is a convex combination of strongly extreme points. We distinguish two cases:

Case I: $i=j$. If $i=j \leq 2$, then let

$$
x_{i, j}^{(l)}=a_{i, j}+(-1)^{l}\left[\left(1-a_{i, j} a_{i, j}^{*}\right)^{\frac{1}{2}} e_{j, j+1}+(i-1) e_{1,1}+\sum_{k=i+1}^{m-1} e_{k, k+1}\right],
$$

for $l=1,2$. If $i=j>2$, then let

$$
x_{i, j}^{(l)}=a_{i, j}+(-1)^{l}\left[e_{j-1, j}\left(1-a_{i, j}^{*} a_{i, j}\right)^{\frac{1}{2}}+\sum_{k=1}^{i-2} e_{k, k+1}+\sum_{k=i+1}^{m} e_{k, k}\right],
$$

for $l=1,2$.

In both cases one can easily see that $x_{i, j}^{(l)}$ is a partial isometry, so that $\left(x_{i, j}^{(l)}\right)^{*} x_{i, j}^{(l)} \geq$ $1-e(i, j)$ and $x_{i, j}^{(l)}\left(x_{i, j}^{(l)}\right)^{*} \geq e(i, j)$ for some $e(i, j) \in$ Lat $\mathcal{A}_{n}$ and $l=1,2$. Thus, an argument similar to the ones in Lemma 6 shows that both $x_{i, j}^{(1)}$ and $x_{i, j}^{(2)}$ are strongly extreme points. The conclusion now follows from the fact that $a_{i, j}=\frac{1}{2}\left(x_{i, j}^{(1)}+x_{i, j}^{(2)}\right)$.

Case II: $i<j$. First notice that $e_{j, i} a_{i, j}$ belongs to the $\mathrm{C}^{*}$-algebra $e_{j, j} \mathrm{C}^{*}(\mathfrak{A}) e_{j, j}$, and thus the Russo-Dye Theorem shows that $e_{j, i} a_{i, j}$ is a convex combination of unitaries from $e_{j, j} \mathrm{C}^{*}(\mathfrak{A}) e_{j, j}$. Hence, $a_{i, j}$ is a convex combination of partial isometries from $\mathrm{C}^{*}(\mathfrak{A})$ with initial spaces equal to $e_{j, j}$ and final spaces equal to $e_{i, i}$. Thus, without loss of generality we may assume that $a_{i, j}$ is such a partial isometry.

If $i>\frac{m}{2}-1$ and $j<\frac{m}{2}+1$, let $b_{i, j}$ be a partial isometry with initial and final spaces

$$
\left[\left\{e_{k, k}: \frac{m}{2}-1<k \leq m, k \neq i\right\}\right] \quad \text { and } \quad\left[\left\{e_{k, k}: 1 \leq k<\frac{m}{2}+1, k \neq j\right\}\right],
$$

respectively. For $l=1,2$, let $x_{i, j}^{(l)}=a_{i, j}+(-1)^{l} b_{i, j}$. In any other case, let

$$
x_{i, j}^{(l)}=a_{i, j}+(-1)^{l} \sum_{\substack{k=1-i \\ k \neq 0}}^{m-j} e_{i+k, j+k} .
$$

In both cases, arguments similar to the ones in Lemma 6 show that $x_{i, j}^{(l)}, l=1,2$, is a strongly extreme point. Moreover, $a_{i, j}=\frac{1}{2}\left(x_{i, j}^{(1)}+x_{i, j}^{(2)}\right)$, and so the proof of Case II is complete.

Theorem 10. Let $\mathfrak{A}$ be a full nest algebra and let $A$ be an element of the open unit ball of $\mathfrak{A}$. Then $A$ is a convex combination of strongly extreme points from $\mathfrak{A}_{1}$.

Proof. Assume that $A$ does not belong to co $\left(\operatorname{s-ext}\left(\mathfrak{A}_{1}\right)\right)$. A standard separation result states that there exist a non-zero linear functional $\varphi$ and a real number $\lambda$ such that $\operatorname{Re} \varphi(x) \leq \lambda \leq \operatorname{Re} \varphi(A)$ for all $x$ in co $\left(\operatorname{s-ext}\left(\mathfrak{A}_{1}\right)\right)$. Since $\operatorname{s-ext}\left(\mathfrak{A}_{1}\right)$ is closed under multiplication by scalars of modulus 1 , it follows that $|\varphi(x)| \leq \lambda$ for all $x$ in co $\left(\operatorname{s-ext}\left(\mathfrak{A}_{1}\right)\right)$. Lemma 9 says that $\operatorname{co}\left(\operatorname{s-ext}\left(\mathfrak{A}_{1}\right)\right)$ contains an open ball of radius $r>0$; thus $\varphi$ is continuous. By Lemma 8 , co $\left(\operatorname{s-ext}\left(\mathfrak{A}_{1}\right)\right)$ is dense in $\mathfrak{A}_{1}$, and so $\|\varphi\| \leq \lambda$. Since $\varphi$ is non-zero, $\lambda>0$. However,

$$
\lambda \leq \operatorname{Re} \varphi(A) \leq\|\varphi\|\|A\| \leq \lambda\|A\|,
$$

and so $\|A\| \geq 1$, a contradiction. 
Remark. Theorem 7 and Theorem 10 imply that if $\mathfrak{A}$ is a semisimple triangular UHF algebra and $\mathfrak{B}$ is a full nest algebra, then $\mathfrak{A}$ and $\mathfrak{B}$ are not isometrically isomorphic as Banach spaces. Paul Muhly has informed us that this fact also follows from [17, Theorem 1.2].

Recently, Power proved that all refinement algebras are isomorphic as Banach spaces, and all standard algebras are isomorphic as Banach spaces [21]. Are the refinement and standard algebras isomorphic as Banach spaces?

Theorem 10 identifies a large family of triangular UHF algebras that satisfy a Krein-Milman type theorem. Are there other significant families of TUHF algebras for which such a theorem holds? A test case may be the block-standard algebra of [11, Example 2.6]. Using the proof of Lemma 6, one can show that the linear span of the strongly extreme points is dense in the algebra. We conjecture that a Krein-Milman type theorem is valid for the block-standard algebra.

\section{REFERENCES}

1. M. Anoussis and E.G. Katsoulis, Finite rank operators and the geometric structure of nest algebras, preprint, 1995.

2. _ A non-selfadjoint Russo-Dye theorem, Math. Ann. 304 (1996), 685-699. CMP 96:10

3. Compact operators and the geometric structure of $\mathrm{C}^{*}$-algebras, Proc. Amer. Math. Soc. 124 (1996), 2115-2122. MR 96i:46068

4. J. Arazy and B. Solel, Isometries of non-self-adjoint operator algebras, J. Funct. Anal. 90 (1990), 284-305. MR 91c:47085

5. J.A. Cima and J. Thomson, On strong extreme points in $H^{p}$, Duke Math. J. 40 (1973), 529-532. MR 47:7440

6. K.R. Davidson, T.G. Feeman, and A.L. Shields, Extreme points in quotients of operator algebras, Topics in operator theory (I. Gohberg, ed.), Birkhäuser Boston, 1988, pp. 67-91. MR 89k:47066

7. M.M. Day, Normed linear spaces, third ed., Springer-Verlag, New York, 1973. MR 49:9588

8. A.P. Donsig, Semisimple triangular AF algebras, J. Funct. Anal. 111 (1993), 323-349. MR 94b: 46084

9. A.P. Donsig and T.D. Hudson, The lattice of ideals of a triangular AF algebra, J. Funct. Anal. 138 (1996), 1-39. CMP 96:13

10. A. Hopenwasser and J.R. Peters, Full nest algebras, Illinois J. Math. 38 (1994), 501-520. MR 95c: 47047

11. T.D. Hudson, Ideals in triangular AF algebras, Proc. London Math. Soc. (3) 69 (1994), 345376. MR 95d: 46060

12. H. Hudzik, W. Kurc, and M. Wisła, Strongly extreme points in Orlicz function spaces, J. Math. Anal. Appl. 189 (1995), 651-670. MR 96f:46051

13. R.V. Kadison and J.R. Ringrose, Fundamentals of the theory of operator algebras, vol. II, Pure and Applied Mathematics, no. 100, Academic Press, San Diego, CA, 1986. MR 88d:46106

14. R. McGuigan, Strongly extreme points in Banach spaces, Manuscripta Math. 5 (1971), 113122. MR 46:7857

15. R.L. Moore and T.T. Trent, Extreme points of certain operator algebras, Indiana Univ. Math. J. 36 (1987), 645-650. MR 89d:47103

16. Isometries of nest algebras, J. Funct. Anal. 86 (1989), 180-209. MR 90k:47096

17. P.S. Muhly, C. Qiu, and B. Solel, On isometries of operator algebras, J. Funct. Anal. 119 (1994), 138-170. MR 95a:46080

18. J.R. Peters, Y.T. Poon, and B.H. Wagner, Triangular AF algebras, J. Operator Theory 23 (1990), 81-114. MR 91h:46102

19. S.C. Power, Classification of tensor products of triangular operator algebras, Proc. London Math. Soc. (3) 61 (1990), 571-614. MR 92a:47053

20. - Limit algebras, Pitman Research Notes in Mathematics, no. 278, Longman Scientific and Technical, London, 1992. MR 94g:46001 
21. algebras, Israel J. Math. 94 (1996), 93-109. CMP 96:14

22. A.G. Robertson, A note on the unit ball in $\mathrm{C}^{*}$-algebras, Bull. London Math. Soc. 6 (1974), 333-335. MR 50:8095

Department of Mathematics, East Carolina University, Greenville, North Carolina $27858-4353$

E-mail address: tdh@.math.ecu.edu

Department of Mathematics, East Carolina University, Greenville, North Carolina $27858-4353$

E-mail address: makatsov@ecuvm.cis.ecu.edu

Department of Mathematics, Texas A\&M University, College Station, Texas 77843

E-mail address: larson@math.tamu.edu 